\title{
Influencia del carbono y el cromo en el comportamiento tribológico de aleaciones férreas
}

\author{
A. García*, C. Río*, Á. Varela*, S. Naya*, R. Losada*y L. García* \\ Resumen En este estudio se analiza la influencia, en las propiedades tribológicas, del contenido en \\ carbono para distintas aleaciones férreas con similares contenidos en cromo, así como la \\ influencia del cromo cuando el contenido en carbono es semejante. También, se analizó la \\ influencia de estos elementos en propiedades como la dureza y en la microestructura de las \\ aleaciones. Para el estudio tribológico se llevaron a cabo dos ensayos, el ensayo descrito en \\ la norma ASTM G105: Standard Test Method for Conducting Wet Sand / Rubber Wheel \\ Abrasion Test y ensayos de chorreo.
}

Palabras clave Desgaste. Dureza. Microestructura. Cromo. Carbono.

\section{Carbon and chrome influence on tribological behaviour of iron alloys}

\begin{abstract}
This study compares the abrasive wear resistance of a carbon steel, used in the linings of coal mills, and ferrous alloys with different chromium and carbon content. It also analyses the influence of chromium and carbon content on mechanical properties, such as hardness, and microstructure. The abrasive wear resistance of the different materials was determined by means of ASTM G 105, "Standard Test Method for Conducting Wet Sand /Rubber Wheel Abrasion Test". A blasting test was used to study erosive wear resistance.
\end{abstract}

Keywords

Wear. Hardness. Microstructure. Chromium. Carbon.

\section{INTRODUCCIÓN}

Uno de los problemas, durante la molienda del carbón, en las centrales térmicas que emplean este material como materia prima consiste en los fenómenos de desgaste que experimentan las distintas partes de los molinos.

Dentro de los molinos de carbón hay dos fenómenos de desgaste que se deben considerar: el desgaste abrasivo y el desgaste erosivo. El primero, se produce como consecuencia de la pérdida de material en la superficie, a causa de la acción cortante de partículas duras, mientras que el segundo se asocia al efecto del impacto de partículas sólidas sobre la superficie ${ }^{[1}$ y 2$]$.

La respuesta de los materiales frente al desgaste, abrasivo o erosivo, está íntimamente relacionada con su microestructura y con sus características mecánicas ${ }^{[3]}$.

Tradicionalmente, se ha empleado como revestimiento de estos molinos acero al carbono y, en la actualidad, las aleaciones férreas con alto contenido en cromo se barajan como alternativa, dadas sus características tanto mecánicas como frente al desgaste $^{[4-6]}$. De esta forma, se pretende conseguir un aumento notable en la durabilidad de estos revestimientos, disminuyendo así los costes ocasionados por el desgaste ${ }^{[7-9]}$.

\section{MATERIALES Y PROCEDIMIENTO EXPERIMEN- TAL}

El estudio se ha enfocado desde dos puntos de vista. Por una parte, se ha comparado el comportamiento presentado por un acero al carbono (empleado tradicionalmente en revestimientos de molinos de carbón) con dos aleaciones férreas con alto contenido en cromo; y, por otra, se ha estudiado la influencia del carbono en la resistencia al desgaste, cuando el contenido en cromo se mantiene.

(*) Escuela Politécnica Superior. Universidade da Coruña. C/Mendizábal s/n, 15403 Ferrol. 


\subsection{Determinación de la composición química}

La composición de las muestras estudiadas se ha determinado a través de dos procedimientos. Mediante absorción atómica se estableció el porcentaje de cromo y molibdeno de cada una de las muestras, y con un analizador de azufre y carbono se determinó el contenido en carbono de las mismas.

\subsection{Determinación de la dureza}

La dureza de las distintas aleaciones se determinó en la escala Vickers, con una carga aplicada de 980 N.

\subsection{Análisis metalográfico}

Para la observación microscópica de la microestructura de las distintas muestras se preparó la superficie de las mismas mediante sucesivas etapas de lijado y pulido, procediéndose a continuación al ataque con $\mathrm{Nital}\left(\mathrm{NO}_{3} \mathrm{H} / \mathrm{CH}_{3} \mathrm{CH}_{2} \mathrm{OH}\right) 5 \%$.

\subsection{Estudio tribológico}

\subsubsection{Determinación de la resistencia al desgas- te abrasivo}

Para determinar la resistencia al desgaste abrasivo de las muestras se siguieron las directrices de la norma ASTM G105 ${ }^{[10]}$. El ensayo definido en esta norma consiste en someter al material a un proceso de desgaste controlado, determinando la pérdida de masa que experimenta como consecuencia de la abrasión.

Las probetas normalizadas son prismas rectangulares con dimensiones 0,025 $\mathrm{m}$ de ancho, 0,057 $\mathrm{m}$ de largo y un espesor de $0,015 \mathrm{~m}$.

Las muestras a ensayar se introducen en una cámara con una mezcla de agua y arena, que actúan como material abrasivo, en proporciones predefinidas $(0,940 \mathrm{~kg}$ de agua y $1,5 \mathrm{~kg}$ de arena). La superficie de las muestras se pone en contacto con la superficie de una rueda giratoria recubierta de caucho. Las dimensiones de la rueda y la dureza de la goma que la recubre están también establecidas en la norma.

El ensayo se realiza en cuatro etapas según el siguiente esquema:

- Primera etapa de preparación. La rueda se recubre con una goma con dureza $50 \pm 2$ Shore A, y gira 1.000 vueltas sobre la superficie de la muestra. El objetivo de esta etapa es preparar la superficie de la muestra.
- Tres etapas de ensayo. En cada una, la rueda gira 1.000 vueltas sobre la superficie ya desgastada en la etapa previa. La dureza de la goma varía de una etapa a otra, siendo de $50 \pm 2$ Shore A, $60 \pm 2$ Shore A y $70 \pm 2$ Shore A, respectivamente. En cada una de las etapas se determina la pérdida de masa experimentada por la probeta a causa de la abrasión.

Una vez determinada la pérdida de masa en cada etapa y normalizados los valores obtenidos para corregir la diferencia entre las dimensiones reales de la rueda y las establecidas en la norma, se representan éstos valores normalizados en función de la dureza de la goma en escala semilogarítmica y se ajusta a una función lineal.

El valor de resistencia al desgaste abrasivo se obtiene como la pérdida de masa que experimenta el material cuando la dureza de la goma que recubre la rueda es, exactamente, 60 Shore A.

\subsubsection{Comportamiento en condiciones de des- gaste erosivo}

Para determinar el comportamiento de los materiales cuando existe desgaste erosivo se utilizó un ensayo de chorreo, consistente en hacer incidir sobre la superficie a ensayar un chorro de aire con partículas duras.

En este caso las probetas ensayadas fueron placas de cada uno de los materiales, con unas dimensiones de 0,2 m de longitud por 0,2 $\mathrm{m}$ de largo y un espesor de 0,015 m.

El chorro impactó sobre cada una de las superficies con una fuerza de $0,49 \mathrm{MPa}$ y un ángulo de $15^{\circ}$, y se mantuvo durante $60 \mathrm{~min}$, de manera que se simularon las condiciones de servicio de los recubrimientos de los molinos de carbón.

Midiendo la pérdida de masa de las probetas a intervalos regulares de tiempo se determinó la velocidad de desgaste a erosión de los materiales estudiados.

\section{RESULTADOS}

\subsection{Composición química}

La tabla I muestra el contenido en carbono, cromo y molibdeno determinado para las muestras.

La muestra $\mathrm{A}$ es un acero al carbono empleado tradicionalmente para la fabricación de placas de revestimiento de los molinos de carbón. 
Tabla I. Porcentaje (en peso) de carbono, cromo y molibdeno de cada muestra

Table I. Carbon, chrome and molybdenum content for each sample

\begin{tabular}{cccc}
\hline Muestra & C (\%) & Cr (\%) & Mo (\%) \\
\hline A & 0,28 & - & - \\
B & 1,05 & 6,0 & 0,55 \\
C & 1,25 & 12,5 & 0,60 \\
D & 1,80 & 12,5 & 0,80 \\
\hline
\end{tabular}

Las muestras B y C presentan contenidos en carbono semejantes, mientras que la principal diferencia entre ellos es el contenido en cromo. La muestra $\mathrm{C}$ presenta, además, un porcentaje en cromo similar al de la muestra $\mathrm{D}$, siendo en este caso, la diferencia fundamental entre las dos aleaciones el contenido en carbono.

\subsection{Valores de dureza}

En la tabla II se encuentran resumidos los valores de dureza Vickers obtenidos para las diferentes aleaciones.

La muestra con un menor valor de dureza es la muestra $\mathrm{A}$, que se corresponde con el acero al carbono empleado en los revestimientos de molinos de carbón. Los materiales alternativos que se proponen (muestras B a D) son aleaciones que presentan valores de dureza mucho más elevados, todos ellos por encima de los $500 \mathrm{HV}$, que se incrementan a medida que aumenta el contenido en carbono y el contenido en cromo.

\subsection{Análisis metalográfico}

La figura 1 muestra la microestructura de la muestra A (Fig. 1a) y de la muestra D (Fig. 1b). En la primera, se observa la estructura típica de un acero al carbono, con granos claros de ferrita y agregados oscuros de perlita, mientras que, en la segunda, es posible apreciar la existencia de carburos precipitados en el borde de grano. La microestructura de

Tabla II. Valores de dureza

Table II. Hardness measurements

\begin{tabular}{lcccc}
\hline Muestra & A & B & C & D \\
\hline Dureza (HV) & 129 & 515 & 613 & 742 \\
\hline
\end{tabular}

Rev. Metal. Madrid Vol. Extr. (2005) 493-497

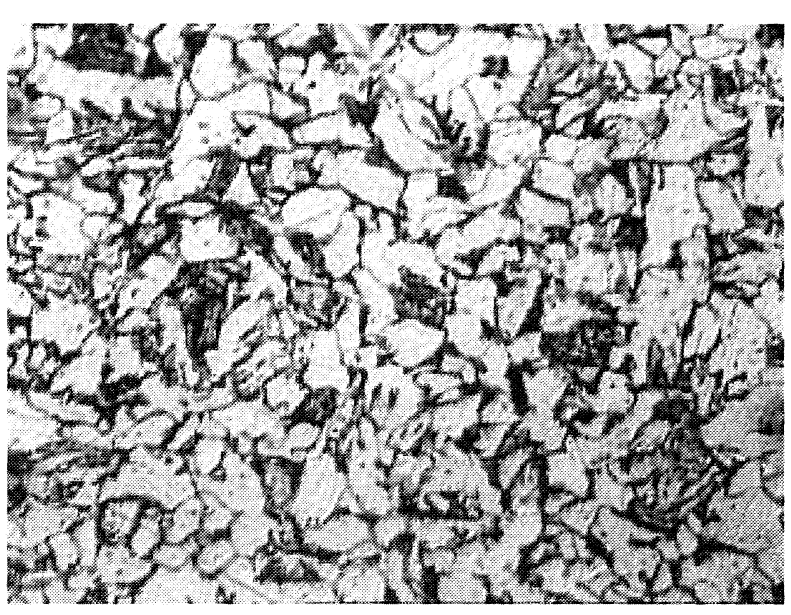

a)

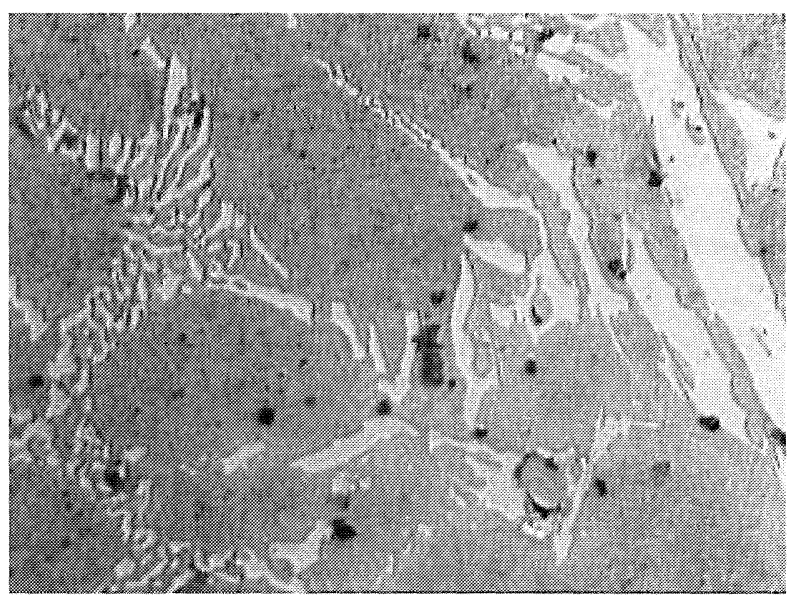

b)

Figura 1. Microestructura de las muestras $A$ y $D$ (micrografías a y $b$ respectivamente) $X 500$.

Figure 1. Samples $A$ and $D$ microstructures (micrograph a and $b$ respectively) $X 500$.

las muestras B, C y D es semejante, siendo la cantidad de carburos precipitados mayor cuanto mayor es el contenido de cromo y carbono.

\subsection{Comportamiento frente al desgaste}

\subsubsection{Resistencia al desgaste abrasivo}

La figura 2 muestra los resultados obtenidos en el ensayo de desgaste abrasivo según la norma ASTM G105, expresados como porcentaje de incremento con respecto a la muestra A, que se toma como referencia por ser el material que se ha empleado en los molinos, tradicionalmente.

En la figura se puede observar como el aumento del contenido de carbono y de cromo, de 0,28 a $1,05 \%$ y de 0 a $6 \%$, respectivamente, implica un 


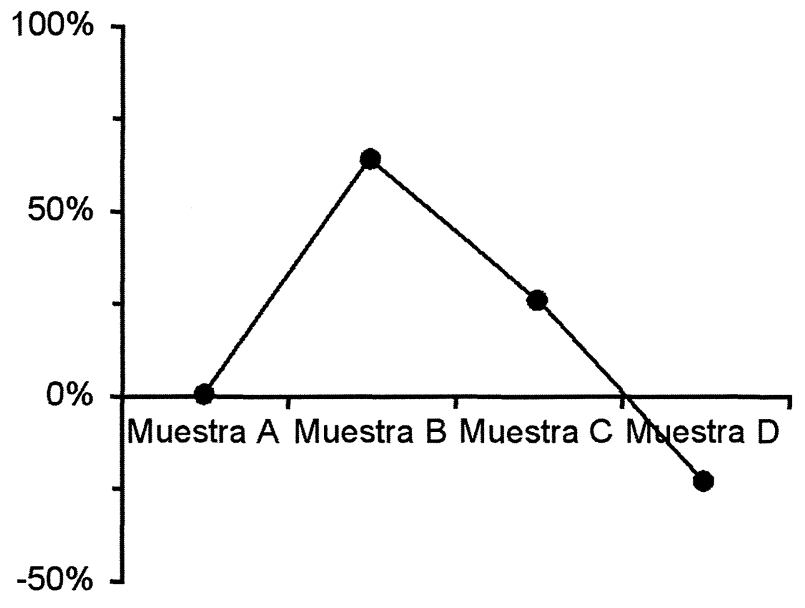

Figura 2. Incremento en el valor de la resistencia al desgaste de las aleaciones con respecto a la muestra $A$.

Figure 2. Increment in abrasive wear resistance values of alloys compared with that of sample $A$.

aumento en la resistencia al desgaste abrasivo, en torno a un $64 \%$. Para la muestra C (con un contenido en cromo del $12,5 \%$ ), el incremento en la resistencia al desgaste abrasivo obtenido es, sin embargo, menor y disminuye aún más para la muestra D que contiene la misma proporción de cromo pero mayor contenido en carbono. Los resultados obtenidos para este último material son, incluso, peores que los obtenidos con el acero al carbono.

\subsubsection{Comportamiento en condiciones de des- gaste erosivo}

En la figura 3 se observa la pérdida de masa experimentada por cada una de las distintas aleaciones durante el ensayo de erosión, medida en intervalos de 20 min. Las muestra B, C y D muestran un desgaste mucho menor que el acero al carbono y su respuesta es prácticamente igual. Se observa, además, que la velocidad de desgaste erosivo se mantiene constante durante el ensayo.

\section{DISCUSIÓN}

El valor de la dureza aumenta cuando el contenido en cromo también lo hace (Tabla II). De esta forma, comparando el valor obtenido para el acero al carbono (muestra A) con el valor de dureza de la aleación con menor contenido en cromo (muestra B) se aprecia un notable aumento de la misma. Cuando se aumenta el contenido en cromo del 6 al $12,5 \%$ (muestra C) el valor de la dureza también aumenta, pero no de una manera tan importante.

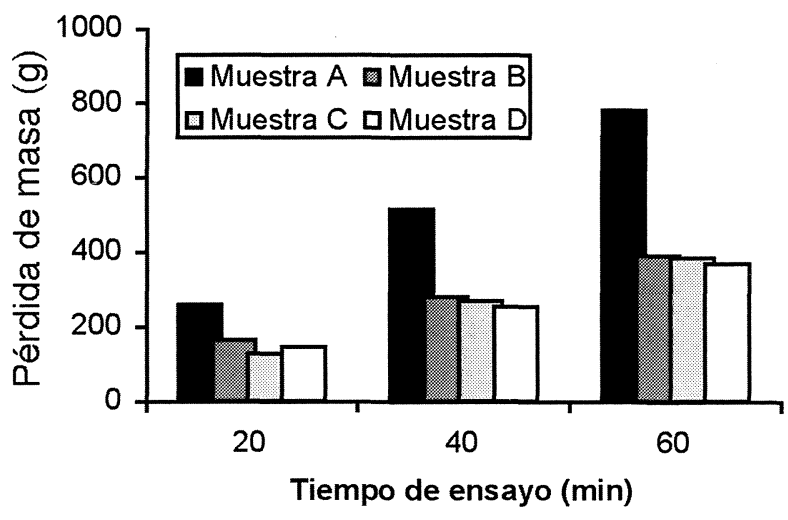

Figura 3. Resultados obtenidos para las distintas muestras en el ensayo de desgaste erosivo.

Figure 3. Erosive wear test results for the different samples.

Lo mismo ocurre cuando se mantiene el contenido en cromo y aumenta el contenido en carbono (muestra D), lo que se produce es un incremento en el valor de la dureza moderado. Según estos resultados, y puesto que la resistencia al desgaste de los materiales está fuertemente relacionada con su dureza, cabría esperar una tendencia similar en los valores obtenidos para los ensayos de desgaste.

En el caso de la resistencia al desgaste abrasivo sí se cumple lo previsto para las muestras A y B, observándose un aumento considerable de este valor cuando se aumenta el contenido en cromo (Fig. 2). Sin embargo, para el resto de las muestras la tendencia se rompe y, a pesar de que la dureza de la muestra $\mathrm{C}$ es mayor que la de la muestra $\mathrm{B}$, el valor de resistencia al desgaste disminuye. Esto puede explicarse si tenemos en cuenta que el aumento en el contenido de cromo propicia la formación de más carburos, de manera que estos carburos se podrían desprender con mayor facilidad de la superficie durante el ensayo, generando mayores pérdidas de peso. Además, el ensayo de determinación de la resistencia al desgaste abrasivo está fuertemente influenciado por las características de la superficie de ensayo, imperfecciones, heterogeneidades...El mismo razonamiento explicaría el resultado obtenido para la muestra $D$ en este ensayo, agravado, aún más, por el mayor contenido en carbono que implicaría una mayor fragilización.

En cuanto al comportamiento frente al desgaste erosivo se aprecia una notable diferencia entre el acero al carbono de la muestra $\mathrm{A}$ y el resto de los materiales ensayados. Las muestras B, C y D presentan resultados muy semejantes, por lo que su comportamiento no varía a pesar de aumentar el contenido en cromo y en carbono. 


\section{CONCLUSIONES}

El aumento del contenido en cromo y del contenido en carbono incrementan el valor de la dureza, observándose un aumento muy importante cuando se compara el acero al carbono de la muestra A con la muestra $\mathrm{B}$ que contiene un $6 \%$ de cromo. $\mathrm{El}$ incremento en la dureza que se consigue duplicando el contenido de cromo o aumentando el carbono no son tan significativos (muestras $\mathrm{C}$ y $\mathrm{D}$ respectivamente).

La resistencia al desgaste abrasivo aumenta de manera importante cuando se añade un $6 \%$ de cromo pero, para contenidos mayores de cromo o carbono, disminuye. El ensayo de resistencia al desgaste abrasivo depende en gran medida de las condiciones superficiales de los materiales a ensayar.

El acero al carbono es el material que presenta una peor respuesta frente a la erosión, siendo el comportamiento mucho mejor cuando se añade cromo. El aumento del contenido en cromo de un 6 a un 12,5\% o el aumento del carbono no supone una mejora del comportamiento frente a la erosión.

\section{REFERENCIAS}

[1] B. BHUSHAN y B.K. GUPTA, Handbook of tribology. Materials, coatings and surface treatments, Krieger Publishing Company, Florida, USA, 1997, pp. 210-229.

[2] E. RABINOWICZ, Friction and wear of materials, John Wiley \& Sons, Inc., Canada, 1995, pp. 191-239.

[3] H. Siera, C.A. García, J. Morales y J.M. Vélez, Revista Universidad EAFIT 117 (2000) 59-67.

[4] J.E. FERnÁndez, R. Vijande, R. TuCho, J. RodríGuez y A. MARTín, Wear 250 (2001) 28-31.

[5] H. Liu, M. Sakamoto, M. Nomura y K. Ogi, Wear 250 (2001) 71-75

[6] W.A. Glaeser, Materials for tribology, Eselvier, Amsterdam, Holanda, 1992, pp. 8-46.

[7] J.A. HAWK, R.D. Wilson, J.H. TYlCZAK y Ö.N. DOGAN, Wear 225-229 (1999) 1.031-1.042.

[8] J.H. TYLCZAK, J.A. HAWK y R.D. WiLsON, Wear 225-229 (1999) 1.059-1.069

[9] G. Muñiz, A. Conde, B.J. Fernández, R. Varela, I. García y J. De Damborenea, Rev. Metal. Madrid 39 (2003) 443-451.

[10] ASTM G105-89, Standards test method for conducting wet sand / rubber wheel abrasion tests, ASTM Standards, pp. 431-439. 